\section{Obesos pero malnutridos: un grave problema en Latinoamérica}

\section{Obese but malnourished: a serious problem in Latin America}

\section{Sr. Editor:}

Dada la gran prevalencia del sobrepeso y la obesidad en las sociedades latinoamericanas, especialmente entre niños y adolescentes ${ }^{1}$, cada vez es más común que el médico, y otros profesionales de la salud, deban atender a pacientes obesos, que padezcan dicho problema como trastorno primario, o presenten dicha condición junto al trastorno principal por el cual son atendidos. Sin embargo, sigue habiendo cierto desconocimiento de las nuevas singularidades con las que en la actualidad se manifiesta clínicamente la obesidad.

Un hecho paradójico que está ocurriendo en muchos países, especialmente en los de ingresos bajos y medios, es la convivencia en la misma población de una alta prevalencia de desnutrición junto a una alta prevalencia de sobrepeso u obesidad. En algunos individuos, sobre todo en niños, pueden coincidir ambas situaciones.

Ya se ha superado la visión que antaño se tenía del niño o del adulto obeso como una persona sana, hipernutrida y feliz. Hoy sabemos que las personas con obesidad pueden estar malnutridas y que suelen tener niveles más altos de psicopatología que las personas en normopeso ${ }^{2}$. Actualmente la mayoría de las personas llegan a la obesidad a través de una alteración continuada del balance energético, en que la ingesta calórica supera al gasto calórico. A ello ha contribuido la modificación de la cantidad y la calidad de los alimentos que se han incluido últimamente en las prácticas de consumo alimentarias, especialmente en poblaciones latinoamericanas ${ }^{3}$. Se observa un progresivo incremento del consumo de alimentos fácilmente disponibles, muy calóricos y más baratos (en comparación con otros más saludables). La alimentación fundamentada en ese tipo de alimentos es un medio que lleva a la obesidad a la vez que a la desnutrición. En este sentido se ha observado en algunos países latinoamericanos que en ciertos hogares de bajos ingresos coexiste la situación de madres con sobrepeso, educando y alimentando a niños que presentan bajo peso y desnutrición ${ }^{4}$.
Algunos de estos nuevos patrones alimentarios son los siguientes:

Por una parte, un cambio en la distribución del consumo de glúcidos, reduciéndose el proveniente de cereales y legumbres (alimentos ricos en fibra, cuyo consumo se asocia a la reducción del riesgo de varias enfermedades), incrementando a la vez el consumo de azúcar, ingrediente que ha pasado a ser clave en muchas bebidas y refrescos, pero también en otros alimentos prefabricados, como las salsas. El abuso del consumo de azúcar se ha considerado como uno de los factores cruciales en la epidemia actual de obesidad, pero también de otros trastornos asociados como la diabetes tipo II o los trastornos cardiovasculares ${ }^{5}$.

Por otra parte, en las últimas décadas también ha sido patente en muchos países latinoamericanos, como Chile 6 , el incremento de la ingesta de grasas saturadas, hasta el punto que en muchas poblaciones un tercio de las calorías diarias provienen de los lípidos. Un exceso de grasas en la alimentación, y especialmente las saturadas, conlleva un incremento del riesgo de obesidad (por su elevado poder calórico), pero también un incremento del riesgo de otras enfermedades, como las cardiovasculares.

La sustitución de una alimentación variada, con una adecuada proporción de macronutrientes (glúcidos, proteínas y lípidos) y de micronutrientes (vitaminas, minerales), alimentación que requiere de cierto poder adquisitivo, por una alimentación centrada en ciertos glúcidos y lípidos, que puede ser más fácil de preparar y de consumir, que produce mayor saciedad, con mayor palatabilidad, y barata, puede ser la explicación de la convivencia en un mismo sujeto de obesidad y malnutrición. A ello, en este panorama pro-obesidad, es necesario añadir que en todas las sociedades se observa una reducción progresiva de la actividad física cotidiana, el incremento del sedentarismo, así como un déficit en la cantidad de agua ingerida diariamente.

Los profesionales de la salud que atienden a un paciente obeso, o que presenta obesidad asociada a otra enfermedad, no deben dar por hecho que están ante un paciente bien nutrido. Dada la situación antes expuesta, cada vez más habitual, el profesional debe cuestionar el estado de salud nutricio del paciente con sobrepeso u obesidad, explorar específicamente esta variable $y$, en su 
caso, proponer una intervención terapéutica específica al respecto.

José I. Baile ${ }^{1, a}$

Facultad de Ciencias de la Salud. Universidad a Distancia de Madrid. Madrid, España. ${ }^{a}$ Doctor en Psicología.

\section{Referencias}

1. Rivera J, González T, Pedraza L, Aburto T, Sánchez, T, Martorell R. Childhood and adolescent overweight and obesity in Latin America: a systematic review. Lancet Diabetes Endocrinol 2013; 70 (13): 173-6.

2. Baile JI, González MJ. Comorbilidad psicopatológica en obesidad. Anales Sis San Navarra 2011; 34 (2): 253-61.

3. Bermúdez OI, Tucker KL. Trends in dietary patterns of Latin American populations. Cad Saude Publica 2003; 19 (Suppl 1): 87-99.

4. Lee JL, Houser RF, Must A, de Fulladolsa PP, Bermúdez OI. Disentangling nutritional factors and household characteristics related to child stunting and maternal overweight in Guatemala. Econ Hum Biol 2010; 8 (2): 188-96.

5. Malik VS, Popkin BM, Bray GA, Després J-P, Hu FB. Sugar Sweetened Beverages, Obesity, Type 2 Diabetes and Cardiovascular Disease risk. Circulation 2010; 121 (11): 1356-64

6. Albala C, Vio F, Kain J, Uauy R. Nutrition transition in Latin America: The case of Chile. Nutr Rev 2001: 59: $170-6$.

Apoyo financiero: Recursos internos de la Universidad a Distancia de Madrid.

Conflictos de intereses: ninguno que declarar.

Correspondencia a:

José I. Baile

Facultad de Ciencias de la Salud, Universidad a Distancia de Madrid.

Carretera de La Coruña, Vía de Servicio, nº 15. KM. 38,500.

Collado-Villalba CP 28400 Madrid-España.

Teléfono: (+34) 918561699

joseignacio.baile@udima.es 